\title{
Operative Behandlung des lumbalen Bandscheibenvorfalls
}

\author{
Andreas Pingel, Matti Scholz, Frank Kandziora
}

\section{Zusammenfassung}

Die operative Versorgung von Bandscheibenvorfällen stellt den am häufigsten vorgenommenen Eingriff an der Wirbelsäule dar. Das Angebot an Verfahren ist mannigfaltig und reicht von perkutaner intradiskaler Laseroder Hitzebehandlung und endoskopischen Techniken bis hin zur mikrochirurgischen Bandscheibenoperation. Obwohl die Morbidität von Bandscheibenoperationen schon recht gering ist, werden immer schonendere operative Verfahren entwickelt und angewendet. Auch wenn nicht jeder Operateur alle Varianten und Zugangsoptionen beherrschen muss, erscheint es sinnvoll, in Abhängigkeit von der Lokalisation des Vorfalls, Beschwerdedauer, anatomischen Gegebenheiten, pathophysiologischen Begleitumständen und Alter des Patienten spezifische, nach Möglichkeit schonende und sichere Operationsverfahren anzuwenden. Die operative Entfernung eines nachgewiesenen Bandscheibenvorfalls bewirkt eine unmittelbare Besserung der radikulären Beschwerden und kann zur Rückbildung neurologischer Ausfälle führen, wenn innerhalb eines von der Schwere der Ausfälle abhängigen Zeitfensters operiert wird.

\section{Operative Treatment of \\ Lumbar Disc Herniation}

The operative treatment of herniated discs is the most commonly performed intervention on the spine. There is a wide variety of procedures ranging from intradiscal percutaneous laser or heat treatment, endoscopic techniques to micro-surgical spine surgery. Although the morbidity of spinal disc surgery is already quite low, less invasive procedures are continuously being developed and applied. Not every surgeon must deal with every possible procedure to treat lumbar herniated discs. Nevertheless, it seems reasonable to choose a specific, gentle and safe surgical access depending on the localisation of the prolapse, duration of complaints, anatomic characteristics, pathophysiological circumstances and age of the patient. The operative removal of a herniated disc brings an immediate improvement in radicular pain and can lead to remission of neurological symptoms, if surgery is performed in an adequate timeframe defined by the severity of symptoms.

\section{Einleitung}

Im Zeitalter zunehmend unphysiologischer Arbeitsbedingungen und Lebensgewohnheiten treten bandscheibenbedingte Erkrankungen in das Leben von immer mehr Menschen. Die verbreitete Bewegungsarmut und Haltungskonstanz in ungünstigen Positionen bewirken

OP-JOURNAL 2009; 25: 112-117

(c) Georg Thieme Verlag KG Stuttgart · New York DOI 10.1055/s-0029-1186061

einen verminderten bewegungsabhängigen Flüssigkeitsaustausch an den Bandscheibengrenzen, der für die Versorgung des bradytrophen Bandscheibengewebes wichtig ist. Dadurch werden Bandscheibendegenerationen begünstigt. Dennoch ist erwiesen, dass anlagebedingte Faktoren eine Schlüsselrolle bei der Entwicklung von Bandscheibenvorfällen spielen $[1,15]$.

Jenseits des 30. Lebensjahrs gibt es fast keinen Menschen mehr, dessen Wirbelsäule nicht schon degenerative Verände- rungen aufweist [6]. Ein Bandscheibenvorfall wird im Allgemeinen zunächst konservativ behandelt. Bei therapieresistenten Beschwerden oder neurologischen Ausfallsymptomen allerdings kann ein chirurgischer Eingriff notwendig werden.

In Deutschland werden im Jahr etwa 70000 Bandscheibenoperationen im Bereich der Lendenwirbelsäule vorgenommen. Damit stellt die Bandscheibenoperation den häufigsten Eingriff an der Wirbelsäule überhaupt dar.

Seitdem in den 30er-Jahren des letzten Jahrhunderts die ersten Bandscheibenoperationen über eine Laminektomie durchgeführt wurden (Mixter und Barr 1934), wurde die Methode nicht zuletzt durch technische Neuerungen wie Lupenbrille, Mikroskop, Endoskopiesysteme sowie Modifikationen der Zugänge entscheidend verändert. Mit der Invasivität wurde auch die Operationsmorbidität minimiert, der Trend geht hin zu gewebeschonenden, wenig traumatisierenden Eingriffen.

Der allgemein anerkannte „Goldstandard" ist nach wie vor in den mikrochirurgischen Operationstechniken zu sehen.

Jedoch werden auch endoskopische Techniken immer häufiger und breiter angewandt und könnten sich in der $\mathrm{Zu}$ kunft aufgrund der minimalen Invasivität mit entsprechend anzunehmendem geringerem Risiko eines Postnukleotomiesyndroms möglicherweise gegenüber den konventionellen Methoden bei der Mehrzahl der zur Operation führenden Befunde durchsetzen.

\section{Indikationen}

Nur etwa $10 \%$ aller nachgewiesenen BSV bedürfen einer operativen Therapie. Indikationen zur primär operativen Behandlung bestehen bei einem Kaudasyndrom (Notfalloperation) und bei signifi- 


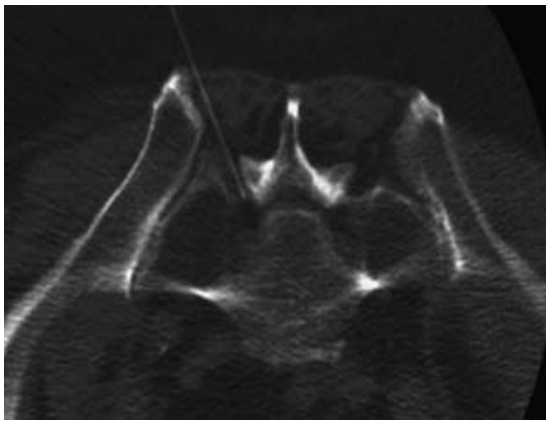

Abb. 1 CT-gesteuerte periradikuläre Infiltration (PRT) der Nervenwurzel S1 links.

kanten und progredienten Paresen (KG 3/5 oder schlechter; dringliche Operationsindikation). Bei massiven radikulären Schmerzen, die kurzfristig nicht durch Analgetika beeinflussbar sind und einem adäquaten Befund in der radiologischen Diagnostik ist ebenfalls ein frühes operatives Vorgehen gerechtfertigt.

In der Mehrzahl der symptomatischen Bandscheibenvorfälle (70-90\%) ist eine konservative Therapie erfolgreich $[2,4]$.

Diese sollte jedoch innerhalb eines Zeitfensters von 6-8 Wochen zu einer signifikanten Linderung der Schmerzen und einer Zunahme der Belastbarkeit führen. Sollte der konservative Behandlungsansatz erfolglos bleiben, müssen mit dem Patienten die operativen Behandlungsoptionen besprochen werden $[10,11]$.

Eine unkritische Fortführung der konservativen Therapiemaßnahmen kann zur Chronifizierung von Schmerzsyndromen führen.

Neben dem objektiven Untersuchungsbefund und den Beschwerden sind noch weitere Kriterien zu berücksichtigen, wie z. B. Dauer und Effektivität der bisherigen Behandlung, Sozialanamnese, Alter und psychische Einstellung des Patienten [6].

\section{Prognose}

Der Erfolg einer operativen Behandlung hängt von einer kritischen Indikationsstellung ab. Sofern bildgebende und klinische Befunde übereinstimmen und eine Korrelation über eine gezielte CTgesteuerte periradikuläre Infiltration (PRT, Abb. 1) noch zusätzlich gesichert werden kann, ist von einer guten Erfolgsaussicht der Sequestrektomie auszugehen.

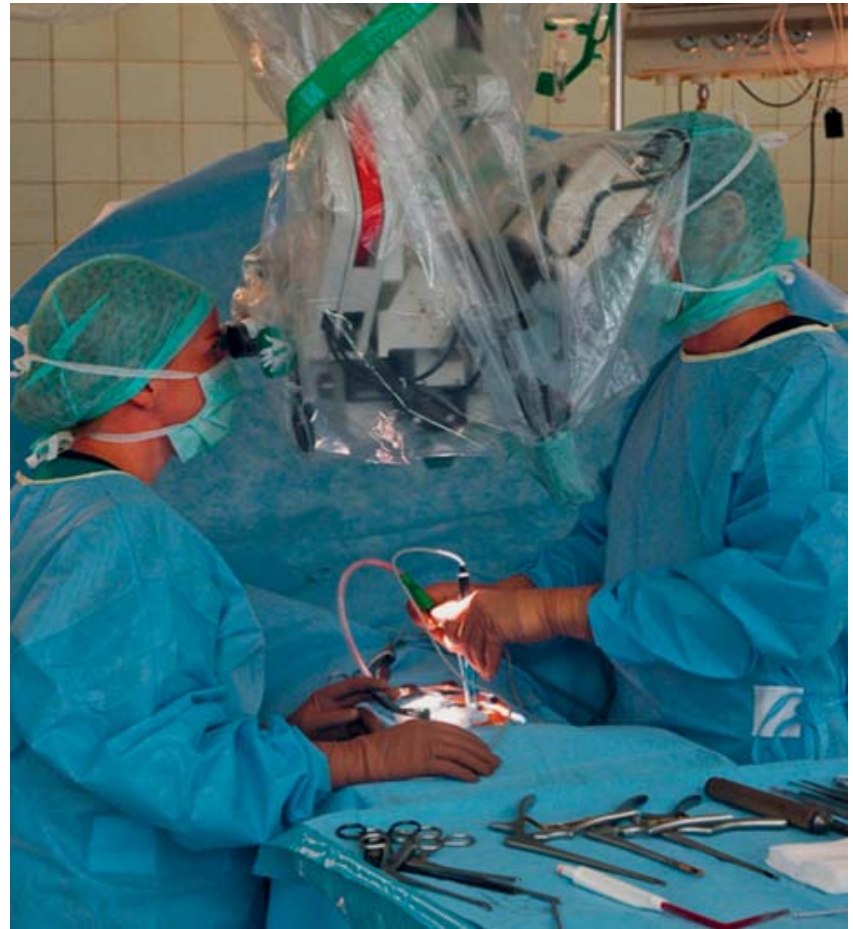

Abb. 2 Mikrochirurgische Bandscheibenoperation.
Hierbei sind positive Prädiktoren eine relativ kurze Beschwerdeanamnese, das Fehlen schwerer neurologischer Defizite, monoradikuläre Syndrome sowie eine sichere berufliche und soziale Situation. Daneben sind ein positives Zeichen nach Lasègue sowie eine Reflexasymmetrie als hinweisend auf den Erfolg einer Bandscheibenoperation anzusehen [10]. Eine Operation führt nachgewiesenermaßen zu einer rascheren Erholung von beeinträchtigenden Schmerzen als die konservative Therapie, auch wenn die Ergebnisse sich im Langzeitverlauf möglicherweise wieder angleichen [4]. Die radikulären Schmerzen sind in aller Regel direkt postoperativ deutlich geringer, die Rückbildung eventueller Sensibilitätsstörungen oder Paresen erfolgt gegenüber dem Spontanverlauf ohne Operation rascher.

Bei über Jahren bestehenden, im Wesentlichen unbeeinflussten chronischen Radikulopathien, insbesondere mit neurologischen Ausfällen, ist die Prognose einer Operation als eingeschränkt einzuschätzen und die Indikation zur Operation entsprechend kritisch zu stellen.

\section{Operative Verfahren}

Es steht eine Vielzahl operativer Behandlungsverfahren zur Verfügung. Allen Operationsmethoden gemeinsam ist das Ziel, das verlagerte Bandscheibengewebe zu entfernen und damit die neuralen Strukturen zu entlasten.
Im Folgenden werden nur die gebräuchlichsten Operationsmethoden genauer aufgeführt. Derzeit werden endoskopische (minimalinvasive) Methoden mit oder ohne Eröffnung des Spinalkanals sowie offene Verfahren angewandt. Bei den offenen Operationen hat sich die mikrochirurgische Technik allgemein durchgesetzt.

\section{Mikrochirurgische Technik}

Die mikrochirurgische Technik ist als „Goldstandard“ die am weitesten verbreitete Methode der Bandscheibenoperation an der Lendenwirbelsäule. Unter Nutzung eines Operationsmikroskops, das neben einer guten Ausleuchtung des OP-Feldes eine bis zu 40-fache Vergrößerung und eine zweidimensionale Darstellung ermöglicht, erfolgt die offene Entfernung des Bandscheibenvorfalls (Abb. 2). Hierbei sind, je nach Lokalisation des Befunds, mehrere Zugangsoptionen möglich.

Bei der mikrochirurgischen Operationstechnik wird häufig eine Hock-BauchLagerung („Häschenstellung“) oder eine Abknickung des Operationstischs mit dem Ziel einer Entlordosierung der Lendenwirbelsäule durchgeführt, um einen besseren interlaminären Zugang zu erhalten. Daneben wird bei der Bauchlagerung auf eine Aussparung des Abdomens geachtet, um den intraabdominellen Druck zu senken und damit das Ausmaß 


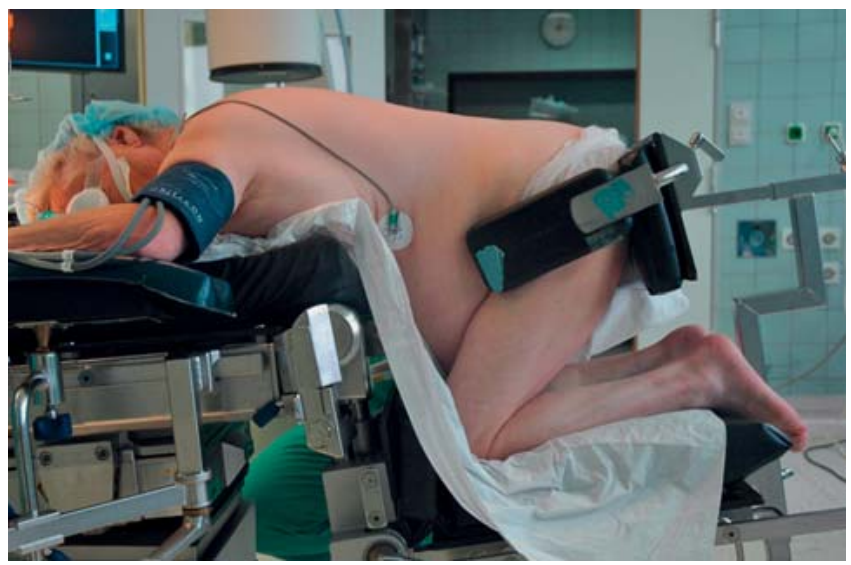

Abb. 3 OP-Setting mit Hock-Bauch-Lagerung („Häschenstellung“) vor mikrochirurgischer Bandscheibenoperation. epiduraler venöser Blutungen zu vermindern (Abb. 3).

\section{Interlaminärer Zugang nach Love (Love und Camp 1937)}

In der Regel (70\%) wird der interlaminäre Zugang angewendet, über den alle intraspinalen Bandscheibenvorfälle erreicht werden können.

Hierbei erfolgen ein medianer/paramedianer Schnitt auf der Seite des Bandscheibenvorfalls, eine Fasziotomie, hiernach das subperiostale Ablösen der Paraspinalmuskulatur, das Darstellen der Wirbelbögen. Nach mikrochirurgischer Resektion des Lig. flavum und ggf. Laminotomie kranial oder kaudal lassen sich der Duralsack und die betroffene Nervenwurzel darstellen, die in der Regel durch den Prolaps verlagert und komprimiert erscheinen. Der Bandscheibenvorfall wird aufgesucht und mittels Häkchen mobilisiert und mit Fasszangen entfernt. Über eine Epiduroskopie wird nach weiteren freien Sequestern gesucht. Der Bandscheibenraum bleibt unangetastet, sofern keine breite Perforation des Anulus fibrosus aufgefunden wird. Blutungen aus epiduralen Venen werden gestillt.

Zur Erhaltung der Stabilität des betroffenen Bewegungssegments ist es von Vorteil, auf eine Nukleotomie, wenn möglich, zu verzichten, daneben sollte eine Eröffnung des Facettengelenks wegen der resultierenden Destabilisierung vermieden werden.

\section{Translaminärer Zugang}

Bei intraspinal nach kranial sequestrierten Bandscheibenvorfällen mit Kompression der kranial des Bandscheibenraums austretenden Nervenwurzel (Abb.4) direkt am Eintritt in das Neuro- foramen bietet sich der translaminäre Zugang als Alternative zum erweiterten interlaminären Zugang an. Modifizierend zum interlaminären Zugang wird über ein umschriebenes Aufbohren der Hemilamina (ca. $1 \mathrm{~cm}$ Durchmesser) der direkte Zugang zum Bandscheibensequester möglich (Abb.5). Auf einen Abstand von mindestens $3 \mathrm{~mm}$ zum lateralen Rand der Pars interarticularis und zu den Laminarändern ist zu achten [3,9, 14]. Da auf eine Hemilaminektomie und eine Facettotomie verzichtet werden kann, ist die Gefahr einer postoperativen Segmentstabilität gemindert.

\section{Extraforaminaler Zugang (nach Benini und Steinsieder 1991)}

Bei extraspinal sequestrierten Bandscheibenvorfällen mit Kompression der kranialen Nervenwurzel am Ausgang aus dem Neuroforamen kann der mikrochirurgische extraforaminale Zugang angewendet werden. Der Vorteil ist, dass das Wirbelgelenk über diesen Zugang geschont werden kann. Daneben kann die autochthone Rückenmuskulatur durch stumpfe Spaltung in ihrer propriozeptiven Funktion erhalten werden. Über zwei Drittel des Sequesters sollten extraforaminal gelegen sein. Eine Manipulation des Ganglions ist möglichst zu vermeiden, dies kann zu postoperativen, nur schwer zu beeinflussenden brennenden Dysästhesien führen, insbesondere bei L5/S1 [8]. Der Hautschnitt verläuft in der Regel ca. $4 \mathrm{~cm}$ paramedian. Ein wiederholtes intraoperatives Durchleuchten zur Lokalisation von kranialem Pedikel und Bandscheibenraum ist hilfreich.

Die Ergebnisse der mikrochirurgischen Bandscheibenoperationen werden in der Literatur als überwiegend positiv beschrieben. In einer Serie von 100 bandscheibenoperierten Patienten hatten

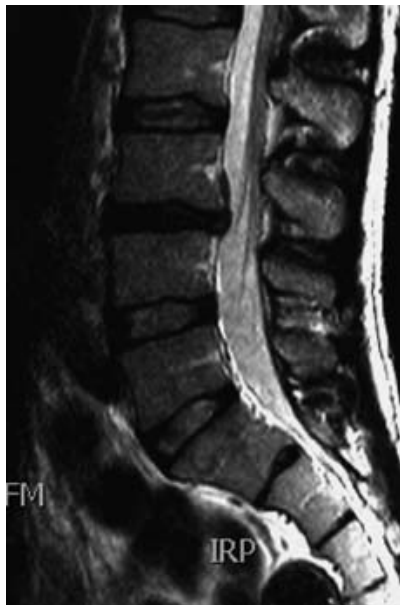

Abb. 4 Nach kranial sequestrierter kleiner BSV L3/ 4 rechts.

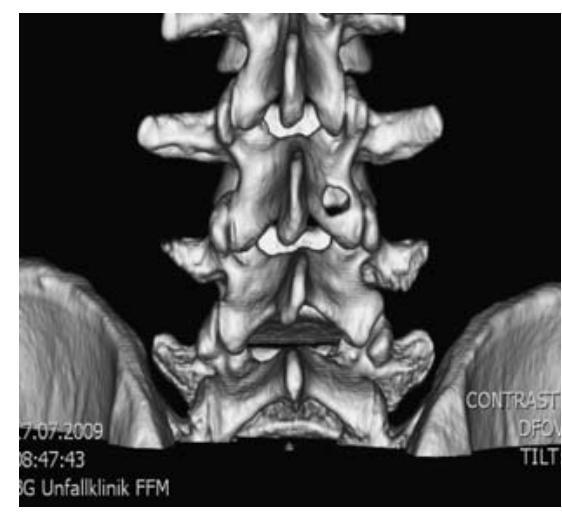

Abb. 5 3D-Rekonstruktion der LWS nach translaminärem Zugang zur Sequestrektomie.

nach einem Jahr 73\% eine komplette Beschwerdefreiheit vonseiten des Beinschmerzes und 63\% eine Beschwerdefreiheit bez. des präoperativ bestehenden Rückenschmerzes. Nach 5-10 Jahren wurden immer noch von 63\% der Patienten eine komplette Beschwerdefreiheit in beiden Kategorien angegeben, 86\% empfanden nach dieser Zeit noch eine Besserung gegenüber dem präoperativen Schmerz, 5\% entwickelten ein „failed back surgery syndrome "[7].

\section{Endoskopische Verfahren}

Der Vorteil der minimalinvasiven Verfahren wird darin gesehen, dass sie bei Umgehung des Wirbelkanals die Morbidität der offenen Bandscheibenoperation reduzieren.

Durch perkutane endoskopische Verfahren ist es möglich, über einen minimalinvasiven Zugang mithilfe eines starren Endoskops unter weitgehender Schonung der Paraspinalmuskulatur frischere Bandscheibenvorfälle zu entfernen. 

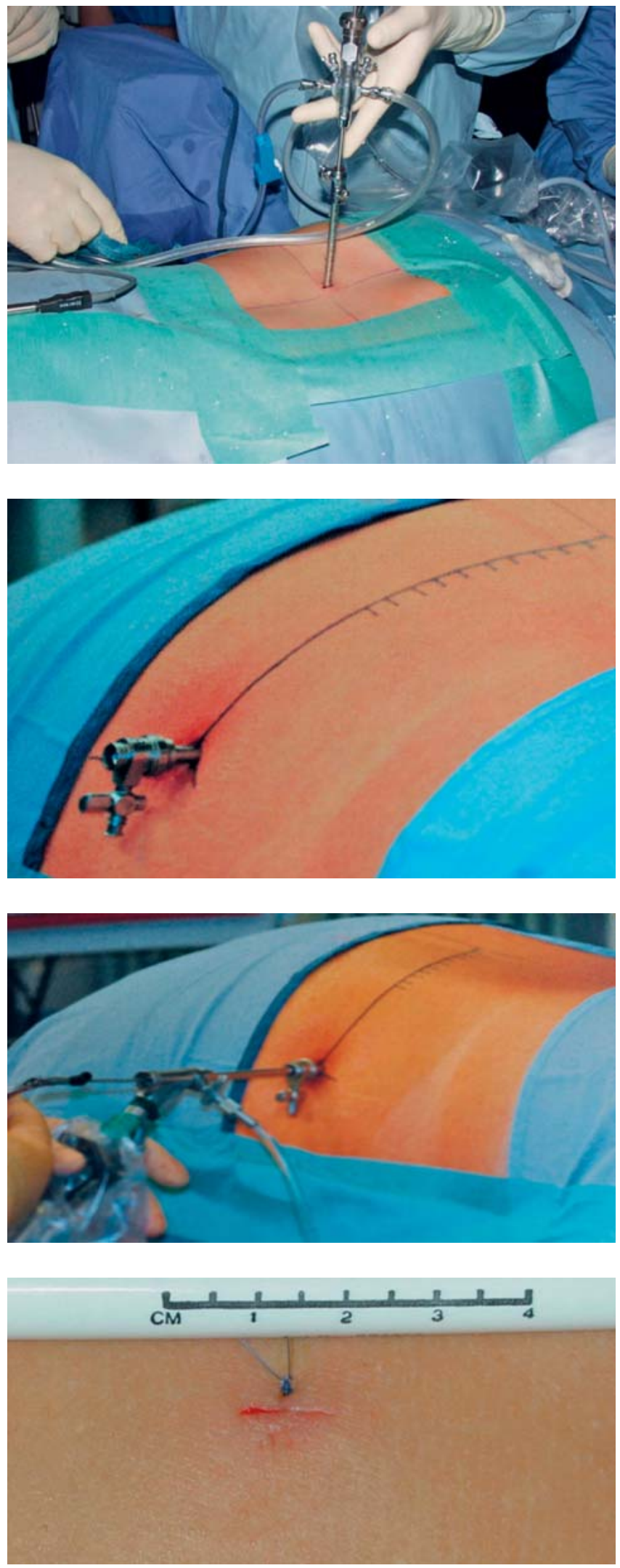

Abb. 6 Endoskopischer interlaminärer Zugang.

Abb. 7 Liegender Arbeitskanal bei lateralem transforaminalen Zugang.

Abb. 8 Endoskopische Sequestrektomie über lateralem transforaminalen Zugang.

Abb. 9 Hautinzision bei endokopischer Bandscheiben-OP.
Zur Lagerung ist eine flache Bauchlage mit Hohllagerung des Abdomens, jedoch ohne Abknickung des Operationstischs zu empfehlen.

Unter Röntgendurchleuchtung erfolgt die Positionierung des Endoskopiesystems. Das Endoskop ist eine Sonde, die mit einer Kaltlichtquelle und einer Kamera ausgestattet ist, deren Bilder auf einen Monitor übertragen werden und so dem Operateur einen Einblick in das Operationsgebiet ermöglicht. Über einen integrierten Arbeitskanal werden verschiedene Instrumente wie Fasszangen, Scheren oder Dissektoren eingebracht, die unter Sicht zur Entfernung des Bandscheibenvorfalls eingesetzt werden können.

Hierbei können, je nach Lokalisation des Befunds, verschiedene Zugänge zur Anwendung kommen.

Beim interlaminären Zugang wird zwischen den Wirbelbögen eingegangen, das Lig. flavum wird gespalten. Hierdurch wird der Zugang zum Spinalkanal ermöglicht (Abb. 6). Zugangsweg und Indikationen des endoskopischen interlaminären Vorgehens unterscheiden sich nicht wesentlich von denen des mikrochirurgischen interlaminären Zugangs.

Beim posterolateralen extraforaminalen Vorgehen wird ein Zugang zum Neuroforamen über einen Winkel von ca. $45^{\circ}$ zur Sagittalebene gewählt.

Beim lateralen transforaminalen Zugang erfolgt ein Zugang zum Spinalkanal transforaminal von streng seitlich (Abb. 7 und 8).

Bei beiden Zugangsvarianten wird transforaminal der Zugang zur intraspinalen oder intraforaminalen Pathologie unter Durchleuchtungskontrolle (Abb. 10 und 11) durchgeführt. Dabei werden die Nervenstrukturen im Wirbelkanal völlig umgangen, somit Verwachsungen und Blutungen vermieden.

Vorteile der endoskopischen Bandscheibenoperation sind in der Minimalisierung des operativen Traumas ohne Störung der Propriozeption mit einer schnelleren Rehabilitation und einem kosmetisch günstigeren Hautschnitt (Abb. 9) zu sehen.

Auch die Risiken einer periduralen Vernarbung oder einer Destabilisierung des Bewegungssegments mit entsprechen- 


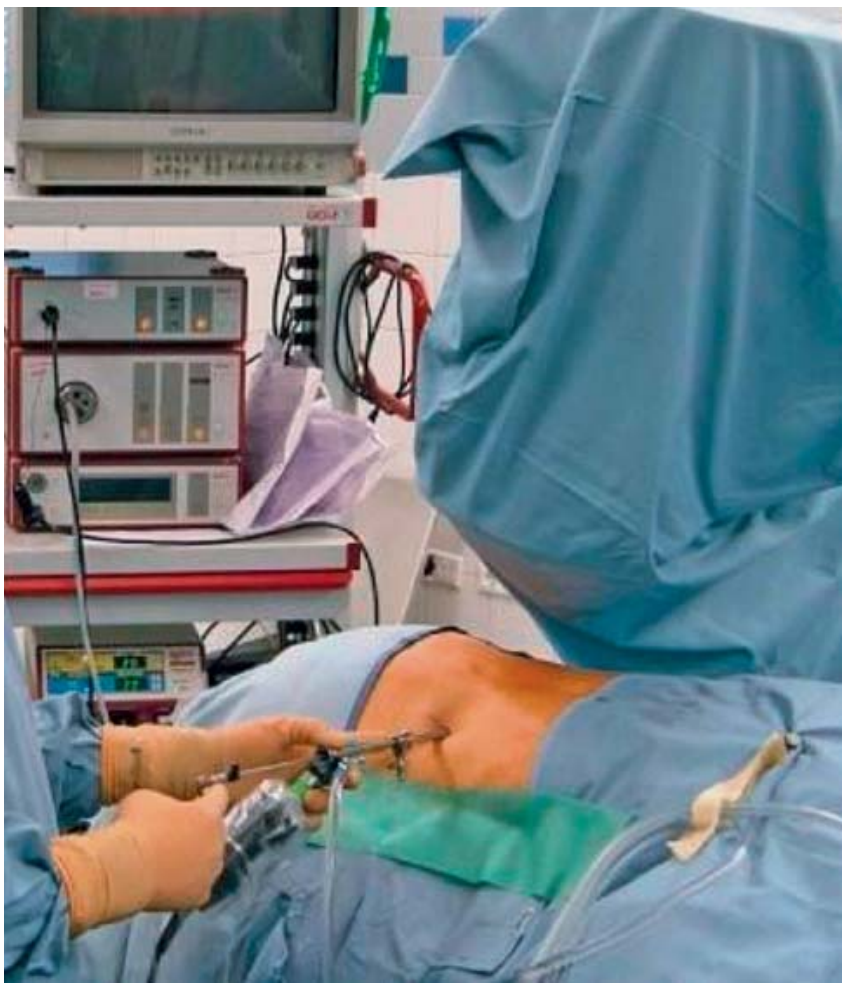

Abb. 10 Intraoperative Durchleuchtungskontrolle des Arbeitskanals. der klinischer Verschlechterung sind hierdurch reduziert. Bei diesem Verfahren ist eine direkte Visualisierung intraund extraforaminaler Vorfälle möglich (Abb. 12-14). Daneben kann der Eingriff bei entsprechender Compliance auch in Lokalanästhesie durchgeführt werden und eignet sich dadurch für eine ambulante Behandlung.

Flexible Hochfrequenz-Bipolarelektroden ermöglichen die Koagulation von Gefäßen direkt an nervalen Strukturen, ohne dass hierdurch Schäden entstehen. Daneben steht eine Vielzahl von Spezialinstrumentarien zur Verfügung. Mittels Shaver (Abb. 12) kann die Nukleotomie erleichtert werden, durch Einsatz von Endoskopiefräsen kann eine Verkleinerung knöcherner Strukturen, etwa bei begleitender Spinalkanalstenose, erfolgen. Die OP-Indikationen unterscheiden sich prinzipiell nicht wesentlich von denen der Standardverfahren. Auch Bandscheibenrezidivoperationen können problemlos durchgeführt werden, da der klassische interlaminäre Zugang zum Spinalkanal umgangen wird.

Nachteilig ist, dass Einschränkungen hinsichtlich der Operabilität bestehen. Das transforaminale Verfahren ist nicht in jedem Fall anwendbar. Bei weit dislozierten Sequestern, bei erheblichen Degenerationen mit foraminaler Stenose $<7 \mathrm{~mm}$ oder einer Rezessusstenose
$<3 \mathrm{~mm}$ ist die Anwendung nicht sinnvoll. Die Anwendung des transforaminalen Zugangs ist zusätzlich limitiert durch die Höhe der Beckenkämme.

Das interlaminäre Verfahren kann nur eingeschränkt angewendet werden bei einem zu kleinen interlaminären Fenster.

Daneben muss mit einem eingeschränkten Blick- und Arbeitsfeld umgegangen werden (Abb. 12-14). Nicht zuletzt die hohen Anschaffungskosten und die erforderlich lange Trainingszeit zum sicheren Erlernen der Technik sind bei der Einführung der endoskopischen Operationsverfahren zu berücksichtigen.

Die Ergebnisse von größeren Studien zur endoskopischen Bandscheibenoperation sind ermutigend und durchaus mit denen der klassischen mikrochirurgischen Technik zu vergleichen.

In einer Untersuchung zu 463 Patienten, die über einen endoskopischen transforaminalen Zugang operiert wurden, wurde bei im Durchschnitt deutlich kürzerer OP-Dauer ( $\varnothing 27 \mathrm{~min}$ ) in einem Nachuntersuchungszeitraum von $1 \mathrm{Jahr}$ keine Komplikation festgestellt, in 7\% kam es zu einem Rezidivprolaps. 81\% der Patienten waren schmerzfrei, bei $88 \%$ bestand Zufriedenheit mit dem Operationsergebnis [13]. Ähnliche Ergebnisse erbrachte eine Untersuchung

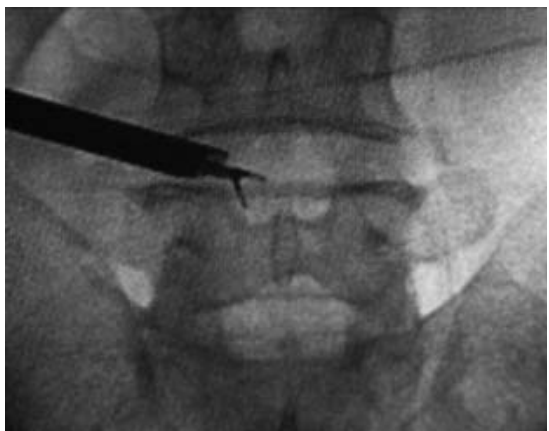

Abb. 11 Intraoperatives Röntgenbild bei lateralem endoskopischen Zugang.

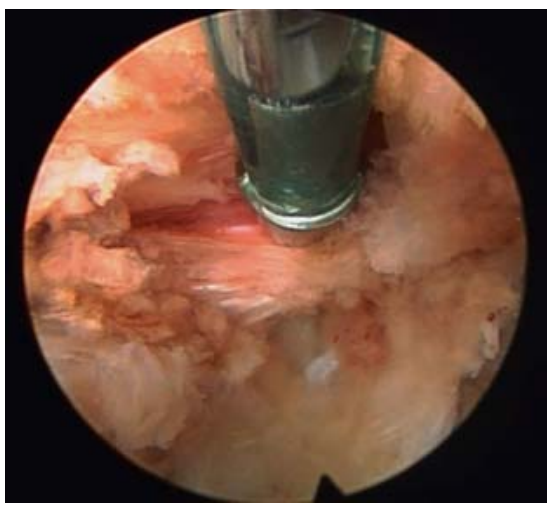

Abb. 12 Entfernen von Teilen des hinteren Längsbands.

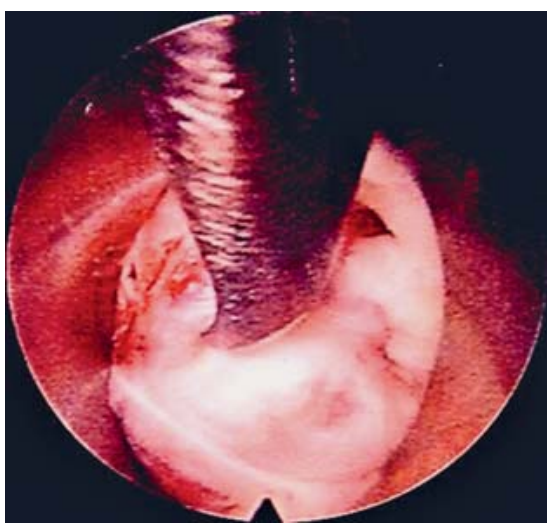

Abb. 13 Mobilisierung eines Bandscheibensequesters.

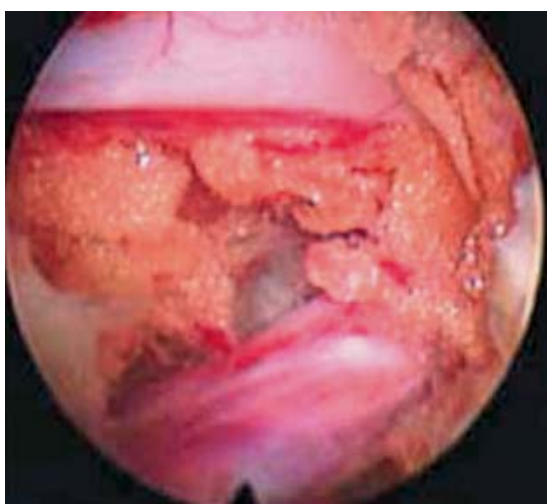

Abb. 14 Darstellen der Wurzelachsel L4/5. 
von 331 Patienten, operiert über einen endoskopischen interlaminären Zugang, die 2 Jahre nachuntersucht wurden. Hier wurden geringe Komplikationen festgestellt $(0,9 \%)$, in nur $2,4 \%$ kam es $\mathrm{zu}$ einem Reprolaps. 82\% der Behandelten waren beschwerdefrei, 91\% waren zufrieden [12].

\section{Komplikationen}

Trotz gewissenhafter Operationsweise ist das Risiko eines Bandscheibenvorfallrezidivs nicht $\mathrm{zu}$ vernachlässigen (3$16 \%)$. Hierbei zeigten neuere Arbeiten keine höheren Rezidivraten bei alleiniger Sequestrektomie gegenüber der Sequestrektomie in Kombination mit gründlicher Nukleotomie [5].

Duraverletzungen sind bei Ersteingriffen an der Bandscheibe relativ seltene Ereignisse (ca. 3\%), bei Revisionsoperationen mit entsprechenden narbigen Verklebungen ist das Risiko jedoch von Relevanz (ca. 15\%). Zum Auftreten einer persistierenden Nervenwurzelschädigung kommt es ebenfalls sehr selten ( $<1 \%)$, ebenso wie zu einer Perforation der ventralen Bandscheibenanteile während der Nukleotomie mit abdominalen Verletzungen $(<0,5 \%)$.

Insbesondere am lumbosakralen Übergang kann es trotz präoperativer Lokalisation mittels Bildwandler zu Segmentverwechslungen kommen. Ein fest eingebauter Röntgenbildverstärker mit der Möglichkeit einer intraoperativen Segmentverifizierung kann dieses Risiko mindern.

Unter dem Begriff „Postnukleotomiesyndrom“ oder besser „failed back surgery syndrome" werden verschiedene pathophysiologische Folgezustände nach Bandscheibenoperationen subsumiert. Hierbei spielt neben einer perineuralen Fibrose („Narbenrezidiv“) auch eine fortschreitende Segmentdegeneration bis hin zur klinisch signifikanten Segmentinstabilität eine Rolle.

Diese erfordern oft Revisionsoperationen mit einer erhöhten Komplikationsgefahr.

Die insbesondere bei begleitender spinaler Enge oft notwendige Resektion knöcherner und ligamentärer Strukturen kann die Stabilität des Segments beeinträchtigen [6].

\section{Fazit}

Bei der Auswahl der operativen Versorgungsverfahren von symptomatischen Bandscheibenvorfällen sollte der spezifischen Situation des Patienten und der Topografie des Prolapses Rechnung getragen werden. Als Goldstandard ist nach wie vor die mikrochirurgische Operationsmethode mit ihren Variationen anzusehen.

Perkutane vollendoskopische Methoden sind in den letzten Jahren perfektioniert worden und ermöglichen bei entsprechenden Indikationen ein schonenderes Vorgehen mit kürzerer Rekonvaleszenz. Jedoch bedarf es noch vergleichender prospektiver Studien zwischen den Ergebnissen der mikrochirurgischen Standardoperation und der Bandscheibenendoskopie, um Stellenwert und Indikationsspektren der operativen Verfahren zu evaluieren.

\section{Literatur}

${ }^{1}$ Beard H, Stevens $R$. Biomechanical changes in the intervertebral disc. In: Jayson M. The lumbar spine and backpain. London: Pitman; 1985

2 Benoist $M$. The natural history of lumbar disc herniations and radiculopathy. Joint Bone Spine 2002; 69: 155-160

3 Di Lorenzo N, Porta F, Omnis G et al. Pars interarticularis fenestration in the treatment of foraminal lumbar disc herniations: a further surgical approach. Neurosurg 1998; 42: 8790

4 Gibson JNA, Grant IC, Waddell G. Surgery for lumbar disc prolapse (Cochrane review). In: The Cochrane library. Issue 1 . Chichester, UK: John Wiley and Sons; 2004
${ }^{5}$ Kast E, Mohr K, Richter HP et al. Ergebnisse der alleinigen Sequestrektomie bei lumbaler Bandscheibenoperation. Orthopäde 2005; 34: 931-937

${ }^{6}$ Krämer J. Bandscheibenbedingte Erkrankungen. Stuttgart, New York: Thieme; 1994

7 Lewis PJ, Weir BKA, Broad R. Long-term prospective study of lumbosacral discectomy. J Neurosurg 1987; 67: 49-53

8 Papavero L. Lumbar disc herniations: the extraforaminal approach. In: Mayer HM, ed. Minimally invasive spine surgery. Heidelberg: Springer; 2005: 297-303

9 Papavero L. Lumbar disc herniations: the translaminar approach. In: Mayer HM, ed. Minimally invasive spine surgery. Heidelberg: Springer; 2005: 304-314

10 Postacchini F. Results of surgery compared with with conservative management for lumbar disc herniations. Spine 1996; 21: 1383-1387

11 Postacchini F, Rothoerl RD, Woertgen C, Brawanski A. When should conservative treatment for lumbar disc herniation be ceased and surgery considered? Neurosurg Rev 2002; 25: 162-165

12 Ruetten S, Komp M, Godolias G. A new full-endoscopic technique for the interlaminar operation of lumbar disc herniations using 6-mm endoscopes: prospective 2-years-results of 331 patients. Minim Invas Neurosurg 2006; 49: 80-87

13 Ruetten S, Komp M, Godolias G. An extreme lateral access for the surgery of lumbar disc herniations inside the sinal canal using the full-endoscopic uniportal transforaminal approach-technique and prospective results of 463 patients. Spine 2005; 30: 2570-2578

14 Soldner F, Helper BM, Wallenfang $T$ et al. The translaminar approach to canalicular and cranio-dorsolateral lumbar disc herniations. Acta Neurochir (Wien) 2002; 144: 315-320

15 Wilson R. Symposium: low back pain and sciatic pain. J Bone Joint Surg [Am] 1968; 50: 1

\section{Dr. med. Andreas Pingel Oberarzt Dr. med. Matti Scholz} Assistenzarzt

Priv.-Doz. Dr. med. Frank Kandziora Chefarzt

Interdisziplinäres Zentrum für Wirbelsäulenchirurgie und Neurotraumatologie

BG-Unfallklinik

Friedberger Landstraße 430

60389 Frankfurt am Main

ws@bgu-frankfurt.de 